\title{
Valuation of Collateralized Funds of Hedge Fund Obligations: a basket option pricing approach
}

Gian Luca Tassinari and Corrado Corradi

\begin{abstract}
The purpose of the present contribution is to provide an extension to a model developed by Tassinari and Corradi [9] to price equity and debt tranches of a Collateralized fund of hedge fund Obligations. Since the value of every tranche depends on the evolution of the collateral portfolio during the life of the contract, the idea is to evaluate each CFO liability as an option on the underlying basket of hedge funds. The proposed model is able to capture skewness, excess-kurtosis in hedge funds' log-returns distribution and to generate a more complex dependence structure than the linear one. At the same time, this new model can be calibrated to the empirical correlation matrix. Finally, the adopted approach allows to find explicit relations among physical and risk neutral processes and distributions at both marginal and joint level.
\end{abstract}

\subsection{Introduction}

In a recent contribution Tassinari and Corradi [9] developed a model to price equity and debt tranches of a Collateralized fund of hedge fund Obligations (CFO). The basic idea developed therein is to compute the fair price of each tranche as its risk neutral expected payoff, discounted at the risk free rate. In fact, as we highlighted, a CFO can be seen as firm with a fixed maturity. Default can be triggered either by the fact that the CFO's Net Asset Value (NAV) at maturity is too low to cover the promised debt payment, as in the traditional Merton's structural firm value model [7], or by the violation of an over collateralization test, which represents a barrier, as

Gian Luca Tassinari

Alma Mater Studiorum Università di Bologna, Dipartimento di Matematica per le Scienze Economiche e Sociali, Viale Filopanti n. 5, Bologna, e-mail: gianluca.tassinari2@unibo.it

Corrado Corradi

Alma Mater Studiorum Università di Bologna, Dipartimento di Matematica per le Scienze Economiche e Sociali, Viale Filopanti n. 5, Bologna, e-mail: corrado.corradi@unibo.it 
in the traditional Black-Cox's model [1]. Thus, each CFO tranche is regarded as an option on the underlying pool of hedge funds. The value of every liability is linked to the dynamics of the collateral portfolio's NAV during the life of the contract. To evaluate dynamically the collateral portfolio NAV it is necessary to model the joint risk neutral evolution of the underlying hedge funds and at the same time any CFO's structural features like coupon payments, over collateralization test, liquidity profile, equity distribution rules, management fees and so on have to be taken into account. In [9] the physical dependence among hedge fund log-returns is introduced through a Gamma stochastic time change of a Multivariate Brownian motion with drift, with independent components [2,6]. From a methodological stand point, the main limitation of that model lies in a low degree of flexibility: in particular, it is not able to replicate the correlations observed in the market. In the present contribution we propose a model able to capture skewness, excess-kurtosis in hedge funds' logreturns distribution and to generate a more complex dependence structure than the linear one. At the same time, this new model can be calibrated to the empirical correlation matrix. Finally, the adopted approach allows to find explicit relations among physical and risk neutral processes and distributions at both marginal and joint level.

The work is organized as follows. In section 1.2 we present the model applied to describe the physical evolution of hedge fund log-returns. In section 1.3 we discuss the change of measure and its impact on marginal and joint processes. In section 1.4 the estimation methodology and the simulation procedure are illustrated. In section 1.5 we discuss the pricing applications and the results.

\subsection{Hedge funds' log-returns P-dynamics}

The dynamics of hedge funds' log-returns is described through a Multivariate Variance Gamma (MVG) process, obtained time changing a Multivariate Brownian motion $(\mathrm{MBm})$, with correlated components, through an independent-common Gamma process. Then, to get more flexibility we added a linear trend. Modelling dependence in this way allows to introduce two sources of co-movement among the NAV of different hedge funds. First, the use of a common stochastic clock introduces a new business time, in which all the market operates, it means all prices jump simultaneously $[2,3,6,9]$. Secondly, jump sizes are correlated $[2,5,8]$. The NAV at time $t$ of each hedge fund is given by the initial NAV times the exponential of a Variance Gamma process with linear drift:

$$
F_{t}^{j}=F_{0}^{j} \exp \left(Y_{t}^{j}\right)
$$

where $F_{t}^{j}$ and $F_{0}^{j}$ is the NAV of the hedge fund $j$ at times $t$ and 0 , while $Y_{t}^{j}$ is the $\log$-return of the $j$-th hedge fund over the interval $[0 ; t]$ for every $j=1, \ldots, n$. The log-return of hedge fund $j$ is 


$$
Y_{t}^{j}=\mu_{j} t+\theta_{j} G_{t}+\sigma_{j} W_{G_{t}}^{j}
$$

where $G=\left\{G_{t}, t \geq 0\right\}$ is the common Gamma stochastic time change process such that $G_{t} \sim \operatorname{Gamma}(t / v, 1 / v)$ and $v>0, W^{j}=\left\{W_{t}^{j}, t \geq 0\right\}$ and $W^{k}=\left\{W_{t}^{k}, t \geq 0\right\}$ are correlated Wiener processes with correlation coefficient $\rho_{j k}, W_{G}^{j}=\left\{W_{G_{t}}^{j}, t \geq 0\right\}$ is the $j$-th Wiener process subordinated by the common Gamma process, $\mu_{j}, \theta_{j}$, and $\sigma_{j}>0$ are constants. The above assumptions lead to the following simple expression for hedge funds' $j$ and $k$ log-returns covariance

$$
\sigma\left(Y_{t}^{j} ; Y_{t}^{k}\right)=\theta_{j} \theta_{k} E\left(G_{t}^{2}\right)+\sigma_{j} \sigma_{k} E\left(G_{t}\right) E\left(W_{t}^{j} W_{t}^{K}\right)=\left(\theta_{j} \theta_{k} v+\sigma_{j} \sigma_{k} \rho_{j k}\right) t
$$

and for correlation

$$
\rho\left(Y_{t}^{j} ; Y_{t}^{k}\right)=\frac{\theta_{j} \theta_{k} v+\sigma_{j} \sigma_{k} \rho_{j k}}{\sqrt{\sigma_{j}^{2}+v \theta_{j}^{2}} \sqrt{\sigma_{k}^{2}+v \theta_{k}^{2}}}
$$

Due to jumps size correlation, this process is more flexible in modelling dependence among hedge funds compared to the one presented in [9]. In particular,

- pairs of hedge funds with skewness of the same sign could be negatively correlated;

- pairs of hedge funds with skewness of opposite sign could be positively correlated;

- an asset with a symmetric distribution could be correlated with other assets;

- pairs of assets have null correlation if and only if at least one of them has a symmetric distribution and their underlying Brownian motions are uncorrelated.

To compute the Characteristic function (Cf) of the MVG process we follow the same procedure of [9]. The Laplace Exponent of the Gamma subordinator is

$$
l(u)=-\frac{\ln (1-u v)}{v}
$$

while the Characteristic Exponent of the MBm with dependent components is

$$
c(\mathbf{u})=\sum_{j=1}^{n} i u_{j} \theta_{j}-\frac{1}{2} \sum_{j}^{n} \sum_{k}^{n} u_{j} u_{k} \sigma_{j} \sigma_{k} \rho_{j k}, \quad \mathbf{u} \in \mathbf{R}^{n} .
$$

Using theorem 4.2 [2] we get the Cf of the MVG process with $t=1$

$$
\begin{aligned}
\Psi_{\mathbf{Y}_{1}}(\mathbf{u})= & \exp \left(i \sum_{j=1}^{n} u_{j} \mu_{j}\right) \times \\
& {\left[1-v\left(\sum_{j=1}^{n} i u_{j} \theta_{j}-\frac{1}{2} \sum_{j}^{n} \sum_{k}^{n} u_{j} u_{k} \sigma_{j} \sigma_{k} \rho_{j k}\right)\right]^{-1 / v} }
\end{aligned}
$$


From this expression we can derive the joint Moment Generating Function (Mgf) of $\mathbf{Y}_{1}$, which is defined when the argument between the square brackets is positive

$$
\begin{aligned}
M_{\mathbf{Y}_{1}}(\mathbf{u})= & \exp \left(\sum_{j=1}^{n} u_{j} \mu_{j}\right) \times \\
& {\left[1-v\left(\sum_{j=1}^{n} u_{j} \theta_{j}+\frac{1}{2} \sum_{j}^{n} \sum_{k}^{n} u_{j} u_{k} \sigma_{j} \sigma_{k} \rho_{j k}\right)\right]^{-1 / v} }
\end{aligned}
$$

From (1.7) its really simple to get the Cf of $Y_{1}^{j}$

$$
\Psi_{Y_{1}^{j}}\left(u_{j}\right)=\exp \left(i u_{j} \mu_{j}\right)\left(1-i u_{j} \theta_{j} v+\frac{1}{2} u_{j}^{2} \sigma_{j}^{2} v\right)^{-1 / v}
$$

\subsection{Change of measure and hedge funds' log-returns $\mathbf{Q}_{\mathbf{h}}-$ dynamics}

Assuming also the existence of a bank account which provides a continuously compounded risk free rate $r$ constant over the interval $[0 ; T]$, our market model is arbitrage free, since the price process of every asset has both positive and negative jumps [2]. This ensures the existence of an equivalent martingale measure. However, the model is not complete, because the risk due to jumps cannot be hedged. Therefore, the equivalent martingale measure is not unique. Among the possible candidates we select the Esscher Equivalent Martingale Measure (EEMM) [4, 8, 9]. The $Q_{\mathbf{h}}$ Esscher measure associated with the multivariate $\log$-returns process $\mathbf{Y}$ is defined by the following Radon-Nikodym derivative:

$$
\frac{d Q_{\mathbf{h}}}{d P} \mid \mathfrak{I}_{t}=\frac{\exp \left(\sum_{j=1}^{n} h_{j} Y_{t}^{j}\right)}{E\left[\exp \left(\sum_{j=1}^{n} h_{j} Y_{t}^{j}\right)\right]}
$$

In order to find the Esscher risk neutral dynamics of $\mathbf{Y}$ two steps are needed:

- find a vector $\mathbf{h}$ such that the discounted price process of every asset is a martingale under the new probability measure $Q_{\mathbf{h}}$, that solves the system

$$
\begin{aligned}
& E\left[\exp \left(\sum_{j=1}^{n} h_{j} Y_{t}^{j}+Y_{t}^{1}\right)\right] / E\left[\exp \left(\sum_{j=1}^{n} h_{j} Y_{t}^{j}\right)\right]=\exp (r t) \\
& \vdots \\
& E\left[\exp \left(\sum_{j=1}^{n} h_{j} Y_{t}^{j}+Y_{t}^{n}\right)\right] / E\left[\exp \left(\sum_{j=1}^{n} h_{j} Y_{t}^{j}\right)\right]=\exp (r t)
\end{aligned}
$$


- find the Cf of the process $\mathbf{Y}$ under $Q_{\mathbf{h}}$.

Making use of (1.8), after some computations and rearrangements, the system (1.11) may be written as:

$$
\begin{gathered}
\frac{1}{v} \ln \left[1-\frac{v\left(\theta_{1}+0.5 \sigma_{1}^{2}+\sum_{j=1}^{n} h_{j} \sigma_{1} \sigma_{j} \rho_{1 j}\right)}{1-v\left(\sum_{j=1}^{n} h_{j} \theta_{j}+\frac{1}{2} \sum_{j}^{n} \sum_{k}^{n} h_{j} h_{k} \sigma_{j} \sigma_{k} \rho_{j k}\right)}\right]=\mu_{1}-r \\
\vdots \\
\frac{1}{v} \ln \left[1-\frac{v\left(\theta_{n}+0.5 \sigma_{n}^{2}+\sum_{j=1}^{n} h_{j} \sigma_{n} \sigma_{j} \rho_{n j}\right)}{1-v\left(\sum_{j=1}^{n} h_{j} \theta_{j}+\frac{1}{2} \sum_{j}^{n} \sum_{k}^{n} h_{j} h_{k} \sigma_{j} \sigma_{k} \rho_{j k}\right)}\right]=\mu_{n}-r
\end{gathered}
$$

with the following constraints

$$
\left[1-v\left(\sum_{j=1}^{n} h_{j} \theta_{j}+\frac{1}{2} \sum_{j}^{n} \sum_{k}^{n} h_{j} h_{k} \sigma_{j} \sigma_{k} \rho_{j k}\right)\right]>0
$$

and

$$
\begin{aligned}
1 & -v\left(\sum_{j \neq q}^{n} h_{j} \theta_{j}+\frac{1}{2} \sum_{j \neq q}^{n} \sum_{k \neq q}^{n} h_{j} h_{k} \sigma_{j} \sigma_{k} \rho_{j k}\right) \\
& -v\left(\left(h_{q}+1\right) \theta_{q}+\frac{1}{2} \sum_{j \neq q}^{n} h_{j}\left(h_{q}+1\right) \sigma_{j} \sigma_{q} \rho_{j q}+\frac{1}{2}\left(h_{q}+1\right)^{2} \sigma_{q}^{2}\right)>0
\end{aligned}
$$

for $q=1, \ldots, n$. From (1.12) we easily get:

$$
\begin{gathered}
\left(a_{1}+\sum_{j=1}^{n} h_{j} b_{1 j}\right) / A_{1}=1-\sum_{j=1}^{n} c_{j} h_{j}-\frac{1}{2} \sum_{j=1}^{n} \sum_{k=1}^{n} h_{j} h_{k} b_{j k} \\
\vdots \\
\left(a_{n}+\sum_{j=1}^{n} h_{j} b_{n j}\right) / A_{n}=1-\sum_{j=1}^{n} c_{j} h_{j}-\frac{1}{2} \sum_{j=1}^{n} \sum_{k=1}^{n} h_{j} h_{k} b_{j k}
\end{gathered}
$$

where $a_{j}=v\left(\theta_{j}+0.5 \sigma_{j}^{2}\right), b_{j k}=v \sigma_{j k}, c_{j}=v \theta_{j}, A_{j}=1-\exp \left[v\left(\mu_{j}-r\right)\right], j=$ $1, \ldots, n$ and $k=1, \ldots ., n$. After some simple rearrangements and computations, the last $n-1$ equations can be written as follows

$$
\begin{gathered}
\sum_{j=2}^{n} h_{j} F_{2 j}=h_{1} D_{2}+E_{2} \\
\vdots \\
\sum_{j=2}^{n} h_{j} F_{n j}=h_{1} D_{n}+E_{n}
\end{gathered}
$$


where $D_{j}=A_{1} b_{j 1}-A_{j} b_{11}, E_{j}=A_{1} a_{j}-A_{j} a_{1}$, and $F_{k j}=A_{k} b_{1 j}-A_{1} b_{k j}$ for $j=$ $2,3, \ldots, n$ and $k=2,3, \ldots, n$. Under the assumption that the matrix of the coefficients $F_{k j}$ is not singular, we can express the solution of (1.16) as a linear function of $h_{1}$, using Cramer's method:

$$
\begin{aligned}
h_{2}= & \frac{\operatorname{det} F_{1}(D)}{\operatorname{det} F} h_{1}+\frac{\operatorname{det} F_{1}(E)}{\operatorname{det} F} \\
& \vdots \\
h_{n} & =\frac{\operatorname{det} F_{n-1}(D)}{\operatorname{det} F} h_{1}+\frac{\operatorname{det} F_{n-1}(E)}{\operatorname{det} F}
\end{aligned}
$$

where $F$ is the coefficients matrix $F_{k j}, F_{k}(D)$ is the matrix obtained substituting its $k$-th column with vector $D, F_{k}(E)$ is the matrix obtained substituting its $k$-th column with vector $E$.

Substituting (1.17) in the first equation of the system (1.15), after tedious computations we get a quadratic equation in only one unknown $h_{1}$ :

$$
e h_{1}^{2}+f h_{1}+g=0
$$

where

$$
\begin{aligned}
& e=\frac{A_{1}}{2} \sum_{k=1}^{n} \sum_{j=1}^{n} I_{k} I_{j} b_{k j} \\
& f=A_{1}\left[\sum_{j=1}^{n} I_{j} c_{j}+\sum_{j=1}^{n} \sum_{k=2}^{n} I_{j} L_{k} b_{j k}\right]+\sum_{j=1}^{n} I_{j} b_{1 j} \\
& g=A_{1}\left[\sum_{j=2}^{n} L_{j} c_{j}+\frac{1}{2} \sum_{j=2}^{n} \sum_{k=2}^{n} L_{j} L_{k} b_{j k}-1\right]+a_{1}+\sum_{j=2}^{n} L_{j} b_{1 j}
\end{aligned}
$$

and with $I_{1}=1, I_{k}=\frac{\operatorname{det} F_{k-1}(D)}{\operatorname{det} F}, L_{k}=\frac{\operatorname{det} F_{k-1}(E)}{\operatorname{det} F}, k=2,3, \ldots n$.

The analysis of the existence of solutions of the equation (1.18), although simple in principle, is a very hard task in practice. However, in all our experiments we found that equation (1.18) possesses a unique solution and therefore a unique vector

$$
\mathbf{h}=\left[h_{1} ; D_{2} h_{1}+E_{2} ; \ldots ; D_{n} h_{1}+E_{n}\right]
$$

exists satisfying the constraints (1.13) and (1.14), where

$$
h_{1}=\frac{-f-\sqrt{f^{2}-4 e g}}{2 e}
$$

This ensures the existence and the uniqueness of the EEMM.

The joint Mgf for $t=1$ under the EEMM can be computed as follows: 


$$
M_{\mathbf{Y}_{1}}^{Q} \mathbf{h}(\mathbf{u})=E^{Q} \mathbf{h}\left[\exp \sum_{j=1}^{n} u_{j} Y_{1}^{j}\right]=\frac{E\left[\exp \sum_{j=1}^{n}\left(h_{j}+u_{j}\right) Y_{1}^{j}\right]}{E\left[\exp \sum_{j=1}^{n} h_{j} Y_{1}^{j}\right]}
$$

After tedious computations and some rearrangements we get the following expression:

$$
\begin{aligned}
M_{\mathbf{Y}_{1}}^{Q} \mathbf{h} & (\mathbf{u})=\exp \left(\sum_{j=1}^{n} u_{j} \mu_{j}\right) \times \\
& {\left[1-\frac{\left.v\left(\sum_{j=1}^{n} u_{j}\left(\theta_{j}+\sum_{k=1}^{n} h_{j} \sigma_{j} \sigma_{k} \rho_{j k}\right)+\frac{1}{2} \sum_{j=1}^{n} \sum_{k=1}^{n} u_{j} u_{k} \sigma_{j} \sigma_{k} \rho_{j k}\right)\right)}{1-v\left(\sum_{j=1}^{n} h_{j} \theta_{j}+\frac{1}{2} \sum_{j}^{n} \sum_{k}^{n} h_{j} h_{k} \sigma_{j} \sigma_{k} \rho_{j k}\right)}\right]^{-1 / v} }
\end{aligned}
$$

The joint $Q_{\mathbf{h}}$ Mgf can be written in the following form

$$
\begin{aligned}
M_{\mathbf{Y}_{1}}^{Q_{\mathbf{h}}} \quad(\mathbf{u}) & =\exp \left(\sum_{j=1}^{n} u_{j} \mu_{j}^{Q_{\mathbf{h}}}\right) \times \\
& {\left[1-v^{Q} \mathbf{h}\left(\sum_{j=1}^{n} u_{j} \theta_{j}^{Q_{\mathbf{h}}}+\frac{1}{2} \sum_{j}^{n} \sum_{k}^{n} u_{j} u_{k} \sigma_{j}^{Q_{\mathbf{h}}} \sigma_{k}^{Q_{\mathbf{h}}} \rho_{j k}^{Q_{\mathbf{h}}}\right)\right]^{-1 / v^{Q} \mathbf{h}} }
\end{aligned}
$$

where relations among statistical and Esscher risk neutral parameters are

$$
\begin{aligned}
\mu_{j}^{Q_{\mathbf{h}}} & =\mu_{j} \\
v^{Q_{\mathbf{h}}} & =v \\
\theta_{j}^{Q_{\mathbf{h}}} & =\frac{\theta_{j}+\sum_{k=1}^{n} h_{k} \sigma_{j} \sigma_{k} \rho_{j k}}{1-v\left(\sum_{j=1}^{n} h_{j} \theta_{j}+\frac{1}{2} \sum_{j}^{n} \sum_{k}^{n} h_{j} h_{k} \sigma_{j} \sigma_{k} \rho_{j k}\right)} \\
\left(\sigma_{j}^{Q} \mathbf{h}\right)^{2} & =\frac{\sigma_{j}^{2}}{1-v\left(\sum_{j=1}^{n} h_{j} \theta_{j}+\frac{1}{2} \sum_{j}^{n} \sum_{k}^{n} h_{j} h_{k} \sigma_{j} \sigma_{k} \rho_{j k}\right)} \\
\sigma_{j k}^{Q_{\mathbf{h}}} & =\frac{\sigma_{j k}}{1-v\left(\sum_{j=1}^{n} h_{j} \theta_{j}+\frac{1}{2} \sum_{j}^{n} \sum_{k}^{n} h_{j} h_{k} \sigma_{j} \sigma_{k} \rho_{j k}\right)} \\
\rho_{j k}^{Q_{\mathbf{h}}} & =\rho_{j k}
\end{aligned}
$$

From (1.26) we can easily obtain the joint $Q_{\mathbf{h}} \mathrm{Cf}$

$$
\begin{aligned}
\left.\Psi_{\mathbf{Y}_{1}}^{Q}{ }_{(\mathbf{u}}\right)= & \exp \left(i \sum_{j=1}^{n} u_{j} \mu_{j}\right) \times \\
& {\left[1-v\left(i \sum_{j=1}^{n} u_{j} \theta_{j}^{Q_{\mathbf{h}}}-\frac{1}{2} \sum_{j}^{n} \sum_{k}^{n} u_{j} u_{k} \sigma_{j}^{Q} \mathbf{h}_{k} \sigma_{k}^{Q} \rho_{j k}\right)\right]^{-1 / v} }
\end{aligned}
$$


The $j$-th $Q_{\mathbf{h}}$ marginal Cf (for $j=1, \ldots, n$ ) is given by:

$$
\Psi_{Y_{1} j}^{Q} Q_{\mathbf{h}}\left(u_{j}\right)=\exp \left(i u_{j} \mu_{j}\right)\left[1-v\left(i u_{j} \theta_{j}^{Q} Q_{\mathbf{h}}-\frac{1}{2} u_{j}^{2}\left(\sigma_{j}^{Q} Q_{\mathbf{h}}\right)^{2}\right)\right]^{(-1 / v)}
$$

Comparing (1.33) and (1.34) with (1.7) and (1.9) it is readily seen that the joint and marginal Cfs under both probability measures are the same type. Under the ESMM the log-returns process is obtained by time-changing a MBm with correlated components, with an independent Gamma process. In particular, the underlying dependence structure is not affected by the change of measure. Precisely, the Brownian motions have the same correlation matrix and the Gamma process has the same parameters under both probability spaces. However, covariances, correlations, and marginal moments change. In particular, the log-return of the $j$-th hedge fund over the interval $[0 ; t]$ under $Q_{\mathbf{h}}$ is

$$
Y_{t}^{j}=\mu_{j} t+\theta_{j}^{Q} \mathbf{h}_{G_{t}}+\sigma_{j}^{Q} \mathbf{h}_{W_{G_{t}}^{j}}
$$

\subsection{Estimation and Simulation}

\subsubsection{Real World and Risk Neutral parameters estimation}

In the applications of section 1.5, the collateral portfolio is made up by eight hedge fund indices, the same reported in $[8,9] .{ }^{1}$ Real world parameters are estimated using a two steps procedure. First step, we estimate the margins using the constrained version of the method of moments described in [8,9]. Second step, we use the implied correlations derived using (1.4) to estimate the correlation matrix. Then, using vector $\mathbf{h}$, the estimates of physical parameters and (1.27), we get the risk neutral ones. This estimation methodology allows to analyse the impact of a different dependence structure comparing the results of this contribution with those of [9]. Tables 1.1 and 1.3 report the marginal parameter estimates and the implied correlation matrix. Esscher risk neutral parameters are reported in Table 1.2. Comparing Table 1.2 with Table 7 of [9], it must be emphasized that the dependence structure has a relevant impact on the risk neutral parameters. In fact, even if marginal parameter estimates are the same under the real world probability measure in both models, under the ESSM they are different. The way, risk premiums are determined, is influenced by the underlying dependence structure. ${ }^{2}$

\footnotetext{
${ }^{1}$ The data set is the same used by [9]

${ }^{2}$ See system (1.12)
} 


\subsubsection{Simulation}

To simulate the paths of $n$ dependent hedge fund NAVs under the EEMM we can proceed as follows.

Let $F_{t_{0}}^{j}$ the NAV of hedge fund $j$ at time 0 for $j=1, \ldots, n$.

Divide the time-interval $[0, T]$ into $N$ equally spaced intervals $\Delta t=T / N$ and set $t_{k}=k \Delta t$, for $k=0, \ldots, N$.

For every hedge fund repeat the following steps for $k$ from 1 to $N$ :

- sample a random number $g_{k}$ out of the $\operatorname{Gamma}(\Delta t / v, 1 / v)$ distribution;

- sample an independent standard Normal random number $w_{t_{k}}^{j}$;

- convert these random numbers $w_{t_{k}}^{j}$ into correlated random numbers $v_{t_{k}}^{j}$ by using the Cholesky decomposition of the implied correlation matrix of the underlying Brownian Motions;

- compute

$$
F_{t_{k}}^{j}=F_{t_{k-1}}^{j} \exp \left[\mu_{j} \Delta t+\theta_{j}^{Q_{h}} g_{k}+\sigma_{j}^{Q_{h}} \sqrt{g_{k}} v_{t_{k}}^{j}\right]
$$

To simulate a simple trajectory of the NAV of the collateral fund of hedge funds it is sufficient to compute for $k$ from 1 to $N$

$$
F_{t_{k}}=\sum_{j=1}^{n} F_{t_{k}}^{j} .
$$

In our applications, we will also take into account the impact of CFO structural features to describe the temporal evolution of the NAV of the collateral portfolio.

\subsection{Applications and Results}

In this section we price debt and equity securities of the three theoretical CFOs described in [9]. We assume the existence of a risk free asset with a constant annual $\log$-return $r=4 \%$. The collateral portfolio is the same in all applications with a CFO a scheduled maturity $T=5$ years. ${ }^{3}$ Firstly, we price a very simple CFO, in which its liability side is represented only by zero coupon bonds with different priorities and an equity tranche. Table 1.4 reports notes and equity fair prices of this CFO. ${ }^{4}$ Secondly, we consider a CFO structure in which liabilities are represented by different coupon bonds and a paying dividend equity tranche. Equity and debt tranches prices are exhibited in Table 1.5. In both previous cases, we have assumed that default could happen only at maturity. In the last application, we introduce the possibility of default before maturity, due to a violation of an over collateralization

\footnotetext{
${ }^{3}$ For a detailed description of these CFO's structures see [9].

${ }^{4}$ The line MVG (IND) contains prices computed using the model developed in [9]. The line MVG (DEP) contains prices computed using the model described in this contribution.
} 
test, and the CFO liquidity profile. Table 1.4 contains our pricing results. ${ }^{5}$ Switching from MVG (IND) to MVG (DEP) model

- without barriers, equity fair prices increase, while lower debt tranches prices decrease

- with barriers, all securities prices decrease.

Results reported in Table 1.4 clearly indicate the importance of modelling risk correctly, both at marginal and joint level. While the price of the equity tranche is marginally affected by the choice of the model, the impact on debt tranches fair prices is really strong.

Table 1.1

Real World Parameters

\begin{tabular}{ccccc}
\multicolumn{5}{c}{ Real World Parameters } \\
\hline Index & $\mu_{j}$ & $\theta_{j}$ & $\sigma_{j}$ & $v$ \\
\hline Convertible Arbitrage & 0,09318 & $-0,02330$ & 0,04590 & 0,33333 \\
Dedicated Short Bias & $-0,05208$ & 0,02691 & 0,16397 & 0,33333 \\
Emerging Markets & 0,13886 & $-0,05419$ & 0,15268 & 0,33333 \\
Equity Market Neutral & 0,08316 & 0,00281 & 0,02647 & 0,33333 \\
Event Driven & 0,17030 & $-0,07013$ & 0,03866 & 0,33333 \\
ED Distressed & 0,17588 & $-0,06401$ & 0,04969 & 0,33333 \\
ED Multi-Strategy & 0,14482 & $-0,05025$ & 0,05321 & 0,33333 \\
ED Risk Arbitrage & 0,08215 & $-0,01534$ & 0,03925 & 0,33333 \\
\hline
\end{tabular}

Table 1.2

Risk Neutral Parameters

\begin{tabular}{ccccc}
\hline Index & $\mu_{j}$ & $\theta_{j}^{Q_{h}}$ & $\sigma_{j}^{Q_{h}}$ & $v$ \\
\hline Convertible Arbitrage & 0,09318 & $-0,05524$ & 0,05619 & 0,33333 \\
Dedicated Short Bias & $-0,05208$ & 0,07054 & 0,20072 & 0,33333 \\
Emerging Markets & 0,13886 & $-0,11797$ & 0,18690 & 0,33333 \\
Equity Market Neutral & 0,08316 & $-0,04400$ & 0,03241 & 0,33333 \\
Event Driven & 0,17030 & $-0,13429$ & 0,04732 & 0,33333 \\
ED Distressed & 0,17588 & $-0,14085$ & 0,06083 & 0,33333 \\
ED Multi-Strategy & 0,14482 & $-0,10879$ & 0,06514 & 0,33333 \\
ED Risk Arbitrage & 0,08215 & $-0,04360$ & 0,04804 & 0,33333 \\
\hline
\end{tabular}

\subsection{Further Reading}

Brooks, C., Kat, H. M., (2002). The Statistical Properties of Hedge Fund Index Returns and Their Implications For Investors. In: The Journal of Alternative Investments, 5 (2), 26-44.

${ }^{5}$ The line MGBm contains prices computed using the multivariate Black and Scholes option pricing model. 
Table 1.3

Brownian Motions Implied Correlation Matrix

\begin{tabular}{ccccccccc}
\hline$\rho_{j k}$ & CA & DSB & EM & EMN & ED & D & MS & RA \\
\hline CA & 1,00 & $-0,27$ & 0,29 & 0,35 & 0,52 & 0,43 & 0,52 & 0,31 \\
DSB & $-0,27$ & 1,00 & $-0,54$ & $-0,34$ & $-0,72$ & $-0,68$ & $-0,57$ & $-0,49$ \\
EM & 0,29 & $-0,54$ & 1,00 & 0,28 & 0,74 & 0,63 & 0,69 & 0,42 \\
EMN & 0,35 & $-0,34$ & 0,28 & 1,00 & 0,53 & 0,47 & 0,41 & 0,30 \\
ED & 0,52 & $-0,72$ & 0,74 & 0,53 & 1,00 & 0,82 & 0,86 & 0,66 \\
D & 0,43 & $-0,68$ & 0,63 & 0,47 & 0,82 & 1,00 & 0,67 & 0,53 \\
MS & 0,52 & $-0,57$ & 0,69 & 0,41 & 0,86 & 0,67 & 1,00 & 0,60 \\
RA & 0,31 & $-0,49$ & 0,42 & 0,30 & 0,66 & 0,53 & 0,60 & 1,00 \\
\hline
\end{tabular}

Table 1.4

Asset Side 1000: Fund of Hedge Funds

Liability Side 1000: Equity and Three Zero Coupon Bonds

\begin{tabular}{ccccc}
\hline Prices & EQUITY & ZCB A & ZCB B & ZCB C \\
& TRANCHE & TRANCHE & TRANCHE & TRANCHE \\
\hline MVG (IND) & 178,641 & 570 & 150,281 & 101,078 \\
MVG (DEP) & 179,036 & 570 & 150,278 & 100,686 \\
\hline
\end{tabular}

Table 1.5

Asset Side 1000: Fund of Hedge Funds

Liability Side 1000: Paying Dividend Equity and Three Coupon Bonds

\begin{tabular}{ccccc}
\hline Prices & EQUITY & CB A & CB B & CB C \\
& TRANCHE TRANCHE TRANCHE TRANCHE \\
\hline MVG (IND) (0\% Div.) & 178,339 & 570 & 150,284 & 101,304 \\
MVG (IND) (50\% Div.) & 178,439 & 570 & 150,276 & 101,165 \\
MVG (IND) (100\% Div.) & 178,623 & 570 & 150,264 & 100,978 \\
\hline MVG (DEP) (0\% Div.) & 178,539 & 570 & 150,268 & 101,134 \\
MVG (DEP) (50\% Div.) & 178,714 & 570 & 150,258 & 100,954 \\
MVG (DEP) (100\% Div.) & 179,009 & 570 & 150,244 & 100,668 \\
\hline
\end{tabular}

\section{Table 1.6}

Asset Side 1000: Fund of Hedge Funds

Liability Side 1000: Paying (50\%) Dividend Equity and Three Coupon Bonds CFO tranche prices with barrier $(105 \%)$ and management fees $(0,5 \%)$

\begin{tabular}{ccccc}
\hline MODEL & EQUITY & CB A & CB B & CB C \\
& \multicolumn{5}{c}{ TRANCHE TRANCHE TRANCHE TRANCHE } \\
\hline $\begin{array}{c}\text { MGBm } \\
\text { Prices with fees }\end{array}$ & 154,977 & 569,912 & 149,994 & 101,439 \\
(Prices with no fees) & $(177,282)$ & $(569,974)$ & $(150,226)$ & $(102,014)$ \\
\hline $\begin{array}{c}\text { MVG (IND) } \\
\text { Prices with fees }\end{array}$ & 154,894 & 567,517 & 146,788 & 92,873 \\
(Prices with no fees) & $(176,443)$ & $(568,073)$ & $(147,725)$ & $(95,291)$ \\
\hline $\begin{array}{c}\text { MVG (DEP) } \\
\text { Prices with fees }\end{array}$ & 154,837 & 566,788 & 145,762 & 90,356 \\
(Prices with no fees) & $(176,199)$ & $(567,475)$ & $(146,921)$ & $(93,294)$ \\
\hline
\end{tabular}


Madan, D. D. and Seneta, E., (1990). The Variance Gamma Model for Share Market Returns. In: Journal of Business, 63 (4), 511-524.

Madan, D. D. and Milne, F., (1991). Option Pricing with V.G. Martingale Components. In: Mathematical Finance, 1 (4), 39-55.

Madan D. D., Carr P. P., Chang, E. C., (1998). The Variance Gamma Process and Option Pricing. In: European Finance Review, 2 (1), 79-105.

Mahadevan, S. and Schwartz, D., (2002). Hedge Fund Collateralized Fund Obligations. In: The Journal of Alternative Investments, 5 (2), 45-62.

Witt G., Fu Y., Leahy J., (2003). Moody's Approach to Rating Collateralized Funds of Hedge Fund Obligations. Moody's Investors Service, 10 June 2003.

Acknowledgements While bearing sole responsibility, the authors gratefully acknowledge useful suggestions by R. Cesari and U. Cherubini.

\section{References}

1. Black F., Cox J. C., (1976). Valuing Corporate Securities: Some Effects on Bond Indenture Provisions. In: Journal of Finance, 31 (2), 351-367.

2. Cont R., Tankov P., (2004). Financial Modelling with Jump Processes. Chapman \& Hall/CRC Press, London.

3. Geman, H., Madan, D. D., Yor, M., (2001). Time Changes for Lévy Processes. In: Mathematical Finance, 11 (1), 79-96.

4. H. U., Shiu, E. S. W., (1994). Option Pricing by Esscher Transforms. In: Transactions of the Society of Actuaries, 46, 99-144.

5. Leoni P., Schoutens W., (2008). Multivariate Smiling. In: Wilmott Magazine, March 2008.

6. Luciano E., Shoutens W., (2006). A Multivariate Jump-Driven Asset Pricing Model. In: Quantitative Finance, 6 (5), 385-402.

7. Merton R., (1974). On the Pricing of Corporate Debt: the Risk Structure of Interest Rates. In: Journal of Finance, 29 (2), 449-470.

8. Tassinari G. L., (2009). Pricing Equity and Debt Tranches of Collateralized Funds of Hedge Fund Obligations. PhD Dissertation, Università di Bergamo. http://aisberg.unibg.it/handle/10446/64.

9. Tassinari G. L., Corradi C., (2011). Pricing Equity and Debt Tranches of Collateralized Funds of Hedge Fund Obligations: an approach based on Stochastic Time Change and Esscher Transformed Martingale Measure. http://amsacta.unibo.it/3256/, (submitted for publication in Quantitative Finance). 